\title{
Effect of thermal vibration and the solid-liquid phase transition on electron dynamics: An inelastic x-ray-scattering study on Al
}

\author{
C. Sternemann, A. Kaprolat, and W. Schülke \\ Institute of Physics, University of Dortmund, D-44221 Dortmund, Germany \\ (Received 16 May 1997; revised manuscript received 2 September 1997)
}

\begin{abstract}
Inelastic-x-ray-scattering measurements of the dynamic structure factor, $S(q, \omega)$, of electrons in Al both in the solid phase at rising temperatures and in the liquid are presented. The double-peak structure of $S(q, \omega)$ for $q>q_{c}\left(q_{c}=\right.$ plasmon cutoff vector), diminishes gradually with decreasing strength of the ion-core potential; the peak position and the sloping plateau of the $S(q, \omega)$ spectra for $q>q_{c}$ do not exhibit marked changes upon melting. The plasmon energy for $q<q_{c}$ shifts according to the variation of the electron density upon heating and melting, and does not respond to the loss of long-range order. [S0163-1829(98)03201-9]
\end{abstract}

The dynamic structure of valence electrons in condensed matter is adequately described in terms of the dynamic structure factor $S(\mathbf{q}, \omega)$, which is the Fourier transform in space and time of the ground-state expectation value of the timedependent density-density correlation operator. ${ }^{1} S(\mathbf{q}, \omega)$ can be measured on an absolute scale by means of inelastic scattering of $\mathrm{x}$ rays $^{2}$ (IXSS) or electrons [electron-energy-loss spectroscopy (EELS)]. ${ }^{3}$ Within the limits of the jellium model, a free-electron gas embedded in a uniform positive background, $S(q, \omega)$ can be calculated in random phase approximation (RPA) by using the well-known Lindhard dielectric function. ${ }^{4}$

But, as far as the intermediate range of momentum transfer $|\mathbf{q}|$, defined by $q_{c}<q<3 p_{F}$, is concerned ( $q_{c}$ is the plasmon cutoff vector, $p_{F}$ is the Fermi momentum), it turned out that the RPA-calculated $S(\mathbf{q}, \omega)$ is far from experiments, performed on $s p$-bound metals like $\mathrm{Li}, \mathrm{Be}$, and $\mathrm{Al} .^{5-10}$ The deviations likewise found for all $s p$-bound metals, are twofold:

(i) The overall shape of the experimental spectra exhibit a main peak, which is shifted to lower energy losses compared with the RPA-calculations. It has been shown in Refs. 7, 9, and 10 that, with respect to the general shape of the spectra, a much better agreement between experiment and calculation could be achieved by taking into account exchange and correlation via local-field corrections. In Refs. 7 and 9 selfenergy corrections have brought calculations still closer to the experiment, though performed only ' on-shell,', a treatment, which has been cast doubt upon by the authors of Ref. 11 , who are claiming an "off-shell", self-energy correction.

(ii) The spectra show some fine structure, which partly depends on the $\mathbf{q}$ orientation, thus proving to be lattice induced, which has been verified by means of pseudopotentialbased model calculations. ${ }^{7,9}$ But there has been found fine structure, which is more or less q orientation independent, ${ }^{7,9}$ and can thus survive averaging in polycrystalline material. It is exactly this type of fine structure, in most cases a doublepeak or a one-peak-one-shoulder structure, which has attracted the attention of theoreticians. There have been made several attempts to interpret this fine structure as a universal property of a correlated electron liquid, either by explaining it as an indication of an incipient Wigner electron lattice ${ }^{5}$ or by introducing damping of the single-particle states via onshell self-energy corrections. ${ }^{12}$ But it must be stressed that a certain amount of theoretical work on that subject failed to reproduce the experimentally found fine structure. ${ }^{13}$ Even self-energy corrections do not yield this fine structure, when performed off-shell. ${ }^{11}$ On the other hand, $a b$ initio bandstructure calculations of $S(\mathbf{q}, \omega)$ on $\mathrm{Al},{ }^{14,15} \mathrm{Be},{ }^{16}$ and $\mathrm{Li},{ }^{17}$ as well as empirical pseudopotential calculations on $\mathrm{Al},{ }^{9}$ have reproduced most of the fine structure in agreement with experiment, in the case of $\mathrm{Al}$ even that type of fine structure which is more or less resistant against orientational averaging.

In spite of this progress at the theoretical front, and since band-structure calculations do not explicitly distinguish between the effect of near-neighbor interaction of atoms in solid and long-range-order effects, it is worthwhile to find out by an experiment, independently of any theoretical model, how the strength of the ion-core potential, on the one hand, and the crystalline long-range order on the other hand, contribute to the dynamic structure factor in the intermediate range of momentum transfer. Therefore, we have measured $S(q, \omega)$ of polycrystalline Al by means of IXSS, where we have gradually diminished the strength of the potential of the long-range-ordered ion cores by heating the Al sample in several steps up to the melting point. Then we have removed the long-range order by investigating $\mathrm{Al}$ in the liquid phase. We found that the general shape of the $S(q, \omega)$ spectra remains unchanged upon heating and melting, so that it cannot be strongly influenced by the crystalline long-range order. Only the dip at $38 \mathrm{eV}$, which characterizes the double-peak structure of the room-temperature $\mathrm{Al}$ spectra, washes out gradually with increasing thermal vibration, disappears nearly upon melting, and must therefore be attributed to the strength of the long-range-ordered ion-core potential.

As far as the range of plasmon excitations $\left(q<q_{c}\right)$ is concerned, most recent calculations of the plasmon dispersion on $\mathrm{Al}$, which include crystal lattice effects as well as (static) local-field corrections, ${ }^{18}$ have revealed good agreement with EELS measurements, ${ }^{19}$ where it is mainly the replacement of jellium by band electrons, which brings the calculations nearer to the experiment. But again, it can be decided only by experiment, whether the removal of long- 

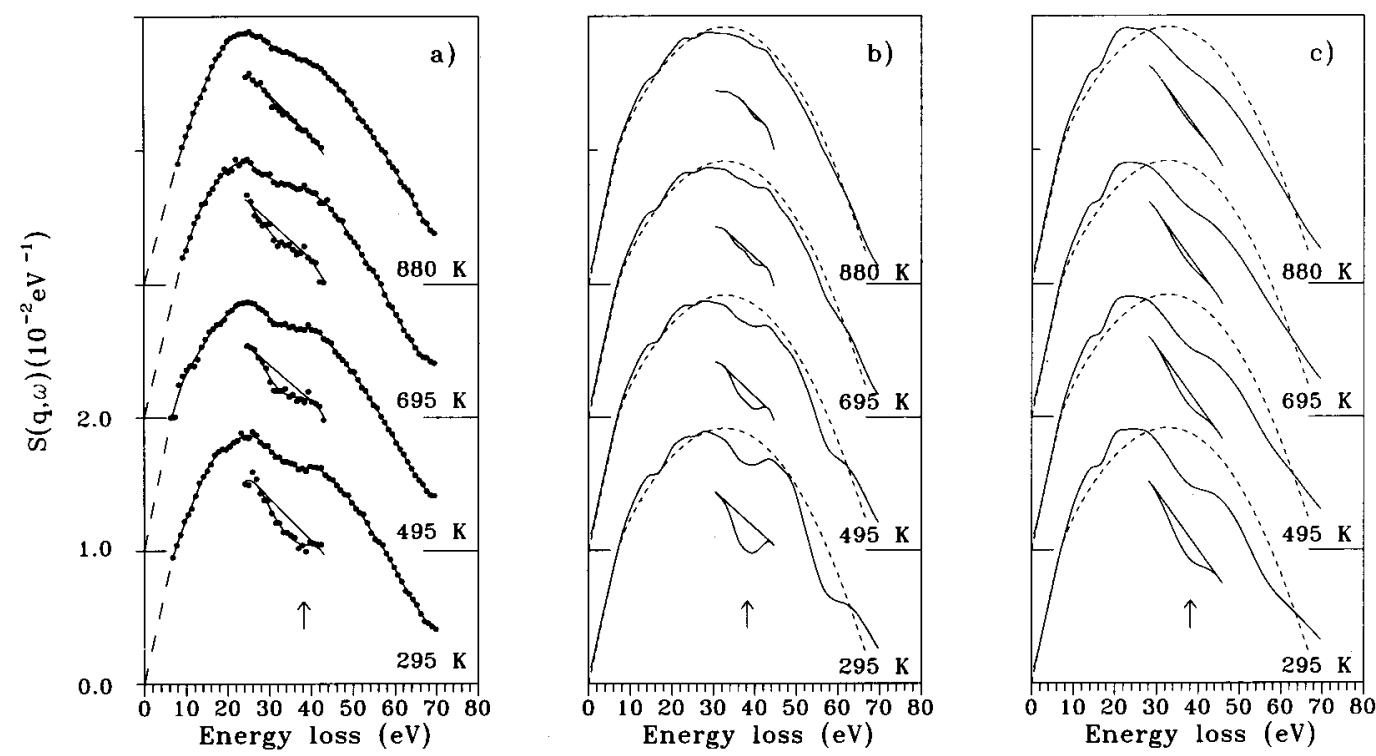

FIG. 1. (a) Data points: Experimental $S(q, \omega)$ for $q=1.5$ at. units of polycrystalline Al at different temperatures as indicated; solid line: cubic spline interpolated experimental data; arrow; position of a zero of the first derivative of the experimental data at $295 \mathrm{~K}$. Insets: Dip region magnified by two. The tangents connect points of equal slope of the interpolated spectra on both sides of the dip. (b) Solid line: Empirical pseudopotential calculated $S(\mathbf{q}, \omega)$ of $\mathrm{Al}$ for $q=1.5$ at. units, weighted average of $\mathbf{q} \|[100]$, [110], [111], [311], and [331] spectra, local-field corrected; temperature effect included via Debye-Waller factor; arrow and insets as in Fig. 1(a). Dashed line: jellium calculated, local-field-corrected $S(q, \omega)$ of Al. (c) Solid line: as in (b), but on-shell-self-energy corrected. Dashed line: (b). Insets: Dip region magnified by 1.5 .

range order upon melting will push the experimental plasmon data back to the calculated jellium data or not, where in the latter case the remaining short-range order could be made responsible for that behavior. We found by measuring the plasmon energy of $\mathrm{Al}$ for a given momentum transfer at rising temperatures below the melting point and in the liquid phase, that no effect of the diminished ion-core potential and of the removed long-range order, respectively, could be detected and that the plasmon energy shift upon heating and melting can be completely explained by the changing of the electron density. ${ }^{20}$

The $S(q, \omega)$ measurements were carried out at the HARWI-inelastic beamline at DESY/HASYLAB. A horizontally focusing $\mathrm{Si}(511)$ monochromator supplies a flux of $3 \times 10^{12}$ photons s ${ }^{-1}$ of $13.7 \mathrm{keV}$ at the sample position. The energy analysis of the scattered radiation is performed in inverse geometry by means of a spherically bent $\mathrm{Si}(12,0,0)$ crystal with a Bragg angle of $86^{\circ}$. By utilizing dispersion compensation $^{7}$ an overall energy resolution of $2.2 \mathrm{eV}$ could be achieved. The 0.5 -mm-thick polycrystalline Al sample with a diameter of $14 \mathrm{~mm}$ is clamped in a ceramic ring and mounted within a $16-\mathrm{cm}$-long and $4-\mathrm{cm}$-wide ceramic tube, which is resistance heated and water cooled outside, so that a temperature up to $1100 \mathrm{~K}$ at the sample position could be attained. The temperature was controlled by a thermocouple mounted very near to the sample position. In the liquid state the thin oxide layer holds the sample in upright position, so that the measurements could be performed in transmission geometry just as in the solid state. The whole scattering chamber is evacuated to $10^{-3}$ mbar. Data were taken at two different scattering angles corresponding to $q=0.385$ at. units (plasmon excitation, temperature steps of $50 \mathrm{~K}$; at. units: $\hbar=e=m=1$ ) and $q=1.5$ at. units (single-particle excitation, temperature steps of $200 \mathrm{~K}$ ), respectively, and were brought to an absolute scale by means of the $f$-sum rule. ${ }^{9}$ The statistical accuracy at the $S(q, \omega)$-peak position was $1 \%$. The subtraction of the quasielastically scattered line, which is increasing in intensity with increasing temperature, makes the data between 0 and $7 \mathrm{eV}$ energy loss less reliable, so that they were skipped in the plots of Fig. 1.

The results of the measurements of $S(q, \omega)$ for $q=1.5$ a.u. in the solid state at different temperatures are plotted in Fig. 1(a). The most prominent fine structure of the experimental spectra, the dip at $38 \mathrm{eV}$, is marked by an arrow that gives the position of a zero of the first derivative of the room-temperature spectrum. This dip is magnified in the inset. A tangent, which connects points of equal slope of the smoothed experimental curve on both sides of the dip, demonstrates its strength. The dip gradually disappears with rising temperature. On the other hand, the overall shape of the spectra shows no dependence on temperature.

The gradual disappearance of the dip is well reproduced by corresponding calculations, which make use of an empirical pseudopotential scheme, and whose details are described in Ref. 9. The theoretical curves, plotted in Fig. 1(b), are the result of a weighted average of $\mathbf{q} \|[100]$, [110], [111], [311], and [331] calculations and are local-field corrected using the static local-field correction factor $G(q)$ from Ref. 21, which has proved to be appropriate for $q<1.5 p_{F} \cdot{ }^{10}$ In order to simulate the influence of the reduced strength of the longrange-ordered ion-core potential due to thermal vibration, we have multiplied the Fourier coefficients $V(\mathbf{G})$ of the empirical pseudopotential ${ }^{22}$ by the corresponding Debye-Waller factor $\exp \left[-B(T)|\mathbf{G}|^{2} / 16 \pi^{2}\right]$, a procedure, which is widely used when band-gap variation with temperature has to be considered in semiconductor physics (see, e.g., Ref. 23). $B(T)$ for Al has been taken from empirical x-ray data of Ref. 
24. The relevant dip region is magnified in the inset. The tangent is defined the same way as for the experimental curves. These calculations reproduce very nicely the experimental findings. This way they deliver an additional proof that it is the diminished strength of the long-range-ordered ion-core potential, which gives rise to the decreasing of the orientation-independent fine structure. It should be stressed that neither in Ref. 14 nor in Ref. 9 could a single Bragg plane be made responsible, in terms of a simple two-band model, ${ }^{7}$ for the more or less orientation-independent dip at $38 \mathrm{eV}$. It is much more the combined action of different Bragg planes, which leads to this fine structure. Additional dips at 16 and $61 \mathrm{eV}$, which appear in the calculations but are not detectable in the experimental curves must be traced back to the action of the (220)-, (440)-, and (333)-Bragg plane, respectively, and are therefore the residues, after orientational averaging, of the very strong structure in the $\mathbf{q} \|[110]$, and $\mathbf{q} \|[111]$ calculation. It is mainly the coarse orientational average, the theoretical curves are based on, and possibly the neglection of lifetime effects, which leads to this difference between calculation and experiment, as far as the dip fine structure is concerned.

Concerning the general shape of the spectra another discrepancy between the results of the empirical pseudopotential calculation and the experiment becomes transparent, especially at higher temperatures. Whereas, with rising temperature, the calculations are approaching the jellium (local-field corrected) result, which is plotted in (b) as a reference, the general shape of the experimental curves at temperatures near the melting point are determined both by a peak position, markedly shifted to lower energy losses compared with the jellium reference, and by the weakly sloping plateau between 25 and $50 \mathrm{eV}$. These deviations could be traced back to inadequacies of our empirical pseudopotential calculation and the way we have handled the temperature effects, above all the neglection of both core orthogonalization and thermal diffuse scattering of the electron state wave functions, where both are mainly determined by the nearneighbor interaction. What is in favor of this explanation is the fact that these discrepancies remain, if one destroys the long-range order by melting, as shown in what follows. It is well known that the short-range order of close-packed materials changes not too much upon melting. ${ }^{25}$

But it is an interesting matter of fact that there exists an alternative explanation for the above-mentioned discrepancy, namely self-energy effects, where the judgement between both types of explanation can be found only by means of a first-principles band-structure calculation of the timedependent local-density approximation quality, which is, as already mentioned, beyond the scope of this communication and which should include the temperature effect in an appropriate way: A much better agreement between the empirical pseudopotential calculation and experiment, especially at higher temperatures, can namely be achieved, if the pseudopotential calculations are on-shell self-energy-corrected by introducing the imaginary part, $\operatorname{Im} \Sigma\left(p, E=p^{2} / 2 m\right)$, of the self-energy into the energy denominator of the Lindhard dielectric function (see Ref. 9). The self-energy used is calculated on the RPA level according to Hedin. ${ }^{26}$ The weakly sloping plateau between 25 and $50 \mathrm{eV}$ is nicely reproduced and the calculated energy position of the main peak coin-

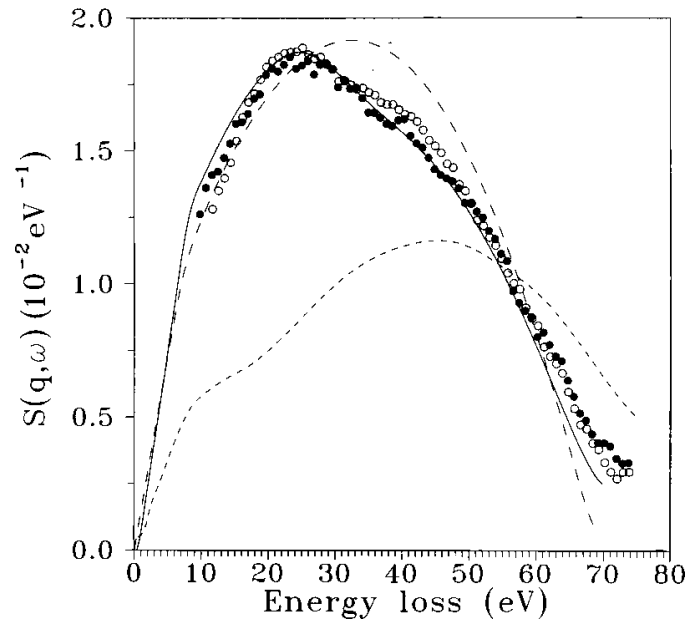

FIG. 2. Solid data points: Experimental $S(q, \omega)$ for $q=1.5$ at. units of liquid $\mathrm{Al}$ at $1000 \mathrm{~K}$; open data points: Experimental $S(q, \omega)$ for $q=1.5$ at. units of polycrystalline $\mathrm{Al}$ at $880 \mathrm{~K}$. Solid line: jellium calculated $\operatorname{Al}-S(q, \omega)$ for $q=1.5$ at. units, on-shell self-energy- and local-field-correction included; long-dashed line: as the solid line but without self-energy correction; short-dashed line: jellium calculated $\mathrm{Al}-S(q, \omega)$ for $q=1.5$ at. units, off-shell self-energy and local-field corrected.

cides with experiment. Moreover, the dips in the pseudopotential calculation at 16 and $61 \mathrm{eV}$ are flattened. We will discuss later, why an on-shell treatment of the self-energy correction might be much more successful than the off-shell treatment, which can claim to be physically more exact. Of course, the two alternative explanations proposed here, represent only the two extremes, so that every position in between might also be possible.

In Fig. 2 the experimental $S(q, \omega)$ spectrum of the liquid phase of $\mathrm{Al}$ at $1000 \mathrm{~K}$ is plotted together with the $880 \mathrm{~K}$ result. The dip at $38 \mathrm{eV}$ has now nearly disappeared, where this vanishing of the fine structure has proved to be reversible, when measuring the same sample again at room temperature after solidification. This behavior must be attributed to the completely destroyed long-range order. But what remains nearly unchanged, when comparing liquid state with solid-state spectra, is their general shape, as far as the peak position and the sloping plateau between 25 and $50 \mathrm{eV}$ are concerned. As already stated for the case of the hightemperature solid-state data, a local-field corrected jellium calculation, as plotted in Fig. 2, does not fit the experimental liquid $\mathrm{Al}$ data. Just as in that case we shall attribute this deviation either to the remaining short-range order in the liquid or to on-shell self-energy effects (or to a combination of both). If the former explanation was valid, the small variation of the general shape upon melting would indicate only small changes of the short-range order induced electronic structure at the phase transition. Such a behavior is in agreement with (i) the (already mentioned) small variation of the short-range order upon melting of close-packed materials, ${ }^{25}$ (ii) the result of investigations of nuclear resonance in solid and liquid metals by Knight and Berger, ${ }^{27}$ (iii) theoretical calculations of the density of states in solid and liquid trivalent elements, ${ }^{28}$ and (iv) theoretical calculations of the optical absorption of simple liquid metals by Sturm and Pajanne. ${ }^{29}$ If the self-energy explanation was valid, the ex- 
perimental data again would indicate that the on-shell approximation works well in the case considered here. In order, to demonstrate how large the difference between on-shell and off-shell self-energy corrections are, we have performed the latter in a way as proposed in Ref. 11, where the modified Lindhard response function includes the full momentum and energy dependence of the self-energy via the corresponding spectral density functions. As shown in Fig. 2, it is surprisingly the on-shell self-energy correction, which fits the experiment very well. The off-shell corrected jellium calculation, however, is far from the experiment. Since it is very unlikely that the very large difference between the off-shell and the liquid $\mathrm{Al}$ data could be removed by properly taking into account the remaining short-range order, this finding is a strong indication for the well-known ${ }^{30,31}$ (partial ?) cancellation between that part of the self-energy correction, which is connected with the reduction of the renormalization constant $z$ (the essence of the off-shell treatment), and the dynamically interacting part of the irreducible particle-hole (vertex) corrections. (Notice that the latter has not been included into our calculation, where only the statically screened particlehole interaction has been taken into account by means of the static local-field correction). A cancellation of this type has been discussed explicitly for the case of optical absorption in Ref. 32 and for the calculation of the dynamical structure factor by Green et al. ${ }^{13,33}$ unfortunately not for the electron density of Al. Further theoretical investigations of this type of cancellation in the case of particle-hole excitations are highly recommended, since they are far beyond the scope of this communication.

It should be stressed that the statements concerning the small changes of the short-range-order-induced electronic properties upon melting would not be falsified, if the selfenergy interpretation of our $S(q, \omega)$ data was correct. On the other hand, our conclusion concerning the cancellation between off-shell self-energy correction and the dynamically interacting part of the vertex correction is hardly influenced by which of the two interpretations of our measurements will turn out to be valid, since all known first-principles band structure calculated $S(q, \omega)$ 's (and these include also the effect of short-range order) exhibit only oscillations around the jellium results (see, for instance, Fig. 1 in Ref. 15), the amplitudes of which are much smaller than the average difference between our on-shell and off-shell calculations.

In Fig. 3 we have plotted the energy position of the $\mathrm{Al}$ plasmon peak $\hbar \omega_{p}(T)$ for $q=0.385$ at. units against temperature, below and above the melting point. Its position at room temperature agrees well with most recent EELS measurements. ${ }^{19}$ We have confronted these measurements with a jellium calculation, which takes into account the dependence of $\hbar \omega_{p}$ on temperature and phase, respectively, only via the electron density $n(T)$ by using the relation $\hbar \omega_{p}(T)=\hbar \omega_{p}\left(T_{0}\right)\left[n(T) / n\left(T_{0}\right)\right]^{1 / 2}$, where the roomtemperature value $\hbar \omega_{p}\left(T_{0}\right)$ is taken from our measurement, the volume expansion coefficients from Ref. 34, and the density variation upon melting $(6.1 \%)$ from Ref. 35 . By comparing calculation and experiment one can conclude that the variation of the plasmon energy with temperature and the magnitude of its discontinuity at the melting point is, within experimental error, solely determined by the corresponding change of the electron density. We have additionally plotted

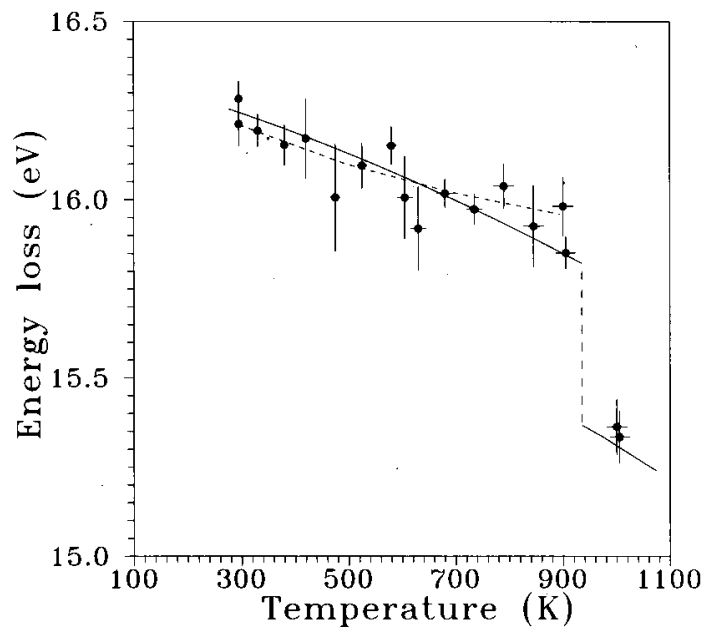

FIG. 3. Data points: Experimental energy position of the plasmon resonance as a function of temperature; solid line: calculated temperature variation of the energy position of the plasmon resonance according to the change of the electron density. Dashed line: relative variation of the plasmon energy position with temperature as calculated within the limits of the empirical pseudopotential scheme including local-field and on-shell self-energy correction. The calculated value at $600 \mathrm{~K}$ is fitted to the experiment.

in Fig. 3 the relative change of the plasmon energy position with raising temperature as obtained by using the above pseudopotential scheme, which multiplies the potential coefficients by Debye-Waller factors in order to mimic the influence of temperature and which takes into account the temperature variation of the electron density. The shift of the plasmon energy position is reduced compared with the simple density-induced shift but only to an extent, which is at the limit to be detectable by experiment. This would mean that diminishing of the strength of the ion-core potential upon heating (and the total removal of the long-range order by melting) has no noticeable influence on the plasmon energy position. Since it is commonly accepted that it is bandstructure-induced interband transition which shift the bandstructure calculated plasmon energy position by more than 1 $\mathrm{eV}$ compare to the jellium value, this insensitivity of the plasmon energy position against removal of the long-range order must be attributed to the persistence of short-range order. ${ }^{25}$ In view of both the rather short wavelength of the plasmons under consideration and the arguments from the literature ${ }^{27-29}$ given above in favor of the short-range order interpretation of the liquid $\mathrm{Al}$ data, the presumed dominance of short-range order is not that surprising. This statement should not contradict a self-energy interpretation of the liquid $\mathrm{Al}$ data in the range of intermediate momentum transfer as long as the deviation of the spectra from jellium due to short-range order cannot be quantified by first-principles calculations, especially since it has been left open whether or not it is self-energy corrections together with the influence of short-range order that are contributing to the liquid Al$S(q, \omega)$.

In summary, our IXSS measurements both on solid $\mathrm{Al}$ as a function of temperature and on $\mathrm{Al}$ in the liquid phase have clearly shown that a nearly q-orientation-independent double-peak fine structure of the dynamic structure factor, $S(q, \omega)$, which has often been considered to be a universal 
property of a strongly correlated electron liquid is longrange-order induced and must be assigned to electron-ion interaction. On the other hand, the overall shape of the Al$S(q, \omega)$ and its deviation from local-field-corrected jellium calculations is hardly influenced by the destruction of the crystalline long-range order. This deviation can either be attributed to the persisting short-range order or to self-energy effects (or to a combined action of both), where arguments are given in favor of performing the self-energy correction on shell. The variation of the plasmon energy with tempera- ture and the discontinuity at the phase transition can be understood as a consequence of the corresponding density changes. The destruction of long-range order has no effect on the plasmon energy. Therefore, the crystal lattice can evolve an influence on the plasmon energy only via the short-range order.

We thank R. Heise, K.-J. Gabriel, K. Höppner, and Ch. Wittkop for valuable help with the measurements. This work was funded by the German Federal Ministry of Education and Research under Contract No. 05 650WEA.
${ }^{1}$ D. Pines and P. Nozieres, The Theory of Quantum Liquids (Addison-Wesley, Reading, MA, 1989), Vol. 1.

${ }^{2}$ W. Schülke, in Handbook on Synchrotron Radiation, edited by G. Brown and D. E. Moncton (North-Holland, Amsterdam, 1991), Vol. 3, p. 609.

${ }^{3} \mathrm{H}$. Raether, in Excitation of Plasmons and Interband Transitions by Electrons, Springer Tracts in Modern Physics Vol. 88 (Springer, Berlin, 1980).

${ }^{4}$ J. Lindhard, K. Dan. Vidensk. Selsk. Mat. Fys. Medd. 28, 1 (1954).

${ }^{5}$ P. M. Platzman and P. Eisenberger, Phys. Rev. Lett. 33, 152 (1974).

${ }^{6}$ W. Schülke and W. Lautner, Phys. Status Solidi B 66, 211 (1974); P. Eisenberger, P. M. Platzman, and P. Schmidt, Phys. Rev. Lett. 34, 18 (1975); A. Vradis and G. D. Priftis, Phys. Rev. B 32, 3556 (1985).

${ }^{7}$ W. Schülke, H. Nagasawa, and S. Mourikis, Phys. Rev. Lett. 52, 2065 (1984); W. Schülke, H. Nagasawa, S. Mourikis, and P. Lanzki, Phys. Rev. B 33, 6744 (1986); W. Schülke, H. Nagasawa, S. Mourikis, and A. Kaprolat, ibid. 40, 12215 (1989).

${ }^{8}$ P. M. Platzman, E. D. Isaacs, H. Williams, P. Zschack, and G. E. Ice, Phys. Rev. B 46, 12943 (1992).

${ }^{9}$ W. Schülke, H. Schulte-Schrepping, and J. R. Schmitz, Phys. Rev. B 47, 12426 (1993).

${ }^{10}$ B. C. Larson, J. Z. Tischler, E. D. Isaacs, P. Zschack, A. Fleszar, and A. G. Eguiluz, Phys. Rev. Lett. 77, 1346 (1996).

${ }^{11}$ G. Niklasson, Solid State Commun. 54, 665 (1985); Tai Kai Ng and B. Dabrowski, Phys. Rev. B 33, 5358 (1986).

${ }^{12}$ G. Mukhopadhyay, R. K. Kalia, and K. S. Singwi, Phys. Rev. Lett. 34, 950 (1975); G. Niklasson, A. Sjölander, and E. Yoshida, J. Phys. Soc. Jpn. 52, 2140 (1983); S. Rahman and G. Vignale, Phys. Rev. B 30, 6951 (1984).

${ }^{13}$ G. Mukhopadhyay and S. Sjölander, Phys. Rev. B 17, 3589 (1978); H. De Raedt and B. De Raedt, Phys. Rev. B 18, 2039 (1978); E. Pajanne, J. Phys. C 15, 5729 (1982); F. Green, D. Neilson, and J. Szymanski, Phys. Rev. 31, 2779 (1985); 31, 2796 (1985); 31, 5837 (1985).
${ }^{14}$ N. E. Maddocks, R. W. Godby, and R. J. Needs, Europhys. Lett. 27, 681 (1994).

${ }^{15}$ A. Fleszar, A. A. Quong, and A. G. Eguiluz, Phys. Rev. Lett. 74, 590 (1995).

${ }^{16}$ N. E. Maddocks, R. W. Godby, and R. J. Needs, Phys. Rev. B 49, 8502 (1994).

${ }^{17}$ H. Bross and M. Ehrnsperger, Z. Phys. B 97, 17 (1995).

${ }^{18}$ A. A. Quong and A. G. Eguiluz, Phys. Rev. Lett. 70, 3955 (1993).

${ }^{19}$ J. Sprösser-Prou, A. vom Felde, and J. Fink, Phys. Rev. B 40, 5799 (1989).

${ }^{20}$ The same conclusion has been drawn for bcc Li by J. P. Hills, C.-C. Kao, W. A. C. Caliebe, D. Gibbs, and J. B. Hastings, Phys. Rev. Lett. 77, 3665 (1996).

${ }^{21}$ K. Utsumi and S. Ichimaru, Phys. Rev. B 22, 5203 (1980).

${ }^{22}$ J. W. D. Connolly, Int. J. Quantum Chem. 35, 807 (1970).

${ }^{23}$ S. Zollner, M. Garriga, J. Humlicek, S. Gopalan, and M. Cardona, Phys. Rev. B 43, 4349 (1991).

${ }^{24}$ R. C. Schukla and C. A. Plint, Phys. Rev. B 40, 10337 (1989).

${ }^{25}$ M. S. Zei and R. Hosemann, Phys. Rev. B 18, 6560 (1978).

${ }^{26}$ L. Hedin, Phys. Rev. 139, A796 (1965).

${ }^{27}$ W. D. Knight and A. G. Berger, Ann. Phys. (N.Y.) 8, 173 (1959).

${ }^{28}$ J. Hafner and W. Jank, Phys. Rev. B 42, 11530 (1990).

${ }^{29}$ K. Sturm and E. Pajanne, J. Phys. F 3, 199 (1973).

${ }^{30}$ K. S. Singwi and M. P. Tosi, Solid State Physics: Advances in Research and Applications, edited by H. Ehrenreich, F. Seitz, and D. Turnbull (Academic, New York, 1981), Vol. 36, p. 177.

${ }^{31}$ G. D. Mahan, Many-Particle Physics (Plenum, New York, 1981), Chap. 6.1.

${ }^{32}$ L. W. Beeferman and H. Ehrenreich, Phys. Rev. B 2, 364 (1970).

${ }^{33}$ F. Green, D. Neilson, and J. Szymanski, Phys. Rev. B 35, 124 (1987).

${ }^{34}$ A. Nicola and M. Domine-Berger, C. R. Acad. Sci. Paris 236, 1021 (1953).

${ }^{35}$ G. Borelius, in Solid State Physics: Advances in Research and Applications, edited by F. Seitz and D. Turnbull (Academic, New York, 1958), Vol. 6, p. 65. 